\title{
Culture-free genotyping of Neisseria gonorrhoeae revealed distinct strains at different anatomical sites in a quarter of patients, the Netherlands, 2012 to 2016
}

Brian MJW van der Veer ${ }^{1}$, Petra FG Wolffs ${ }^{1}$, Christian JPA Hoebe ${ }^{1,2}$, Nicole HTM Dukers-Muijrers ${ }^{1,2}$, Lieke B van Alphen ${ }^{1}$

1. Department of Medical Microbiology, Care and Public Health Research Institute (CAPHRI), Maastricht University Medical Centre (MUMC+), Maastricht, the Netherlands

2. Department of Sexual Health, Infectious Diseases and Environmental Health, South Limburg Public Health Service, Heerlen, the Netherlands

Correspondence: Lieke van Alphen (lieke.van.alphen@mumc.nl)

van der Veer Brian MJW, Wolffs Petra FG, Hoebe Christian JPA, Dukers-Muijrers Nicole HTM, van Alphen Lieke B. Culture-free genotyping of Neisseria gonorrhoeae revealed distinct strains at different anatomical sites in a quarter of patients, the Netherlands, 2012 to 2016. Euro Surveill. 2018;23(50):pii=1800253. https://doi. org/10.2807/1560-7917.ES.2018.23.50.1800253

Background: Genotyping of Neisseria gonorrhoeae (NG) is essential for surveillance to monitor NG transmission and dissemination of resistant strains. Current genotyping methods rely on bacterial culture which frequently fails. Aim: Our aim was to develop a culture-free genotyping method that is compatible with the widely used $N$. gonorrhoeaemulti-antigen sequence typing (NG-MAST) database, which facilitates genotyping of NG detected at separate anatomical sites in individual patients. Methods: Specific primers for both PCR targets por $B$ and $\operatorname{tbp} B$ were designed and technically validated by assessing the analytical sensitivity, cross-reactivity with 32 nongonoccocal Neisseria species, and concordance with NG-MAST. Clinical application was assessed on 205 paired samples from concurrent NG infections at different anatomical sites of 98 patients ( 81 men who have sex with men and 17 women) visiting our sexually transmitted infections clinic. Results: Typing could be consistently performed on samples with a PCR quantification cycle $(\mathrm{Cq})$ value $<35$. Furthermore, the method showed no cross-reactivity and was concordant with NG-MAST. Culture-free NG-MAST improved the typing rate from $62 \%(59 / 95)$ for cultured samples to $94 \%$ (89/95) compared with culture-dependent NG-MAST. Paired samples of 80 of 98 patients were genotyped, revealing distinct NG strains in separate anatomical sites in $25 \%(20 / 80)$ of the patients. Conclusions: This NG-specific genotyping method can improve NG surveillance as it facilitates genotyping of non-culturable and extra-genital samples. Furthermore, 25\% of patients were infected with multiple NG strains, which is missed in current culture-dependent surveillance. Including non-culturable and concurrent NG infections in surveillance informs actions on dissemination of multidrug-resistant NG strains.

\section{Introduction}

Neisseria gonorrhoeae (NG) is one of the most common bacterial sexually transmitted infections [1]. The World Health Organization (WHO) estimates that more than 100 million new cases of NG occur each year, even though testing for NG and diagnostics have improved $[1,2]$. Detection of NG allows empirical treatment that results in cure in at least $95 \%$ of cases, and rapid cure subsequently limits transmission [1]. However, increasingly resistant strains of NG have been reported in the last decades, which could complicate empirical treatment [3]. Therefore, gaining insight in transmission and antimicrobial resistance (AMR) of NG is important. NG can be detected by culture or nucleic acid amplification test (NAAT) but both methods have limitations [2]. Culture is known to be less sensitive because NG requires demanding nutritional and environmental conditions, leading to a low percentage of culture-confirmed diagnoses [4]. In contrast, NAAT are more sensitive but cannot determine the AMR profile $[2,5]$.

Surveillance of NG is essential to monitor transmission and dissemination of resistant strains. NG multiantigen sequence typing (NG-MAST) is a widely used genotyping method to monitor transmission and outbreaks $[6,7]$. This method has a higher discriminatory power than multilocus sequence typing (MLST) and is more cost-effective than highly discriminatory whole genome sequencing [8,9]. In addition, some of the NG-MAST sequence types (ST) are associated with AMR $[7,10]$. The currently used NG-MAST protocol requires culture because the primers cross-react with other Neisseria species [11]. To date, only two studies have genotyped non-cultured clinical samples with NG-MAST $[6,11]$. Whiley et al. demonstrated that NG-MAST can be applied to non-cultured urogenital samples but not to samples from extra-genital sites 
(oropharynx and rectum) because of the presence of commensal Neisseria species [11]. They showed that mainly N. lactamica, N. meningitidis and N. polysaccharea strains lead to the cross-reactivity. Furthermore, it appeared that successful application of NG-MAST to non-cultured samples was linked to the quantification cycle (Cq) of the PCR-positive sample because four of the five failed samples had a high Cq value (>35).

Previous studies have shown that patients can be NG-positive at extra-genital sites and have concurrent NG infections at different anatomical sites $[12,13]$. Most of the extra-genital and concurrent infections are observed in risk groups, for example in men who have sex with men (MSM). Extra-genital sites may act as a reservoir for AMR genes as the present commensal Neisseria species, potentially harbouring AMR genes, readily exchange DNA with NG [14]. Typing the oropharyngeal site using culture-dependent methods is especially difficult because the bacterial load is lower than at other anatomical sites and this appears to be linked to culture success [15]. In previous studies, concurrent NG infections were studied with various genotyping methods [6,11,16-18]. Distinct NG strains per anatomical site have been observed and some strains demonstrated discordant antibiotic susceptibility profiles [6,16-18]. The observed distinct NG strains could be explained by high-risk sexual behaviour and patients being part of different transmission chains $[16,18]$. However, these studies were small (fewer than 10 patients), focussed on cultured isolates, used a single-position single nucleotide polymorphisms (SNP) and/or used non NG-specific primers [6,16-18]. Therefore, we aimed to develop a culture-free NG-MAST genotyping method that does not cross-react with other Neisseria species and is compatible with the NG-MAST database. Furthermore, we aimed to gain more insight in the frequency of distinct NG strains at separate anatomical sites in individual patients.

\section{Methods}

This study was designed to test the clinical application of the culture-free NG-MAST method to non-culturable clinical samples and use these data to compare ST of separate anatomical sites within a patient. The method was technically validated by assessing analytical sensitivity, specificity and concordance with NG-MAST.

\section{Clinical samples}

All NG-positive clinical samples $(n=1,110)$ from different anatomical sites were retrieved from 814 consultations (further referred to as number of patients) from 642 individual patients. NG positivity was based on NG detection by the Cobas 4800 CT/NG NAAT assay (Roche Diagnostics, Basel, Switzerland), between January 2012 and May 2016 from our sexually transmitted infections (STI) clinic (South Limburg Public Health Service). These samples were from MSM ( $n=769$ samples), women ( $n=254$ samples) and heterosexual men $(n=87$ samples). Samples with a $\mathrm{Cq}$ value of 35 or higher did not consistently yield PCR products in dilution series (see technical validation). Therefore, clinical samples with a $\mathrm{Cq}$ value of $\geq 35$ were excluded $(n=418)$, leaving 692 samples for analysis. Of the remaining 692 NG-positive samples, we included only paired samples from separate anatomical sites belonging to a single STI clinic visit of a patient $(n=228)$. Different pairs of any combination of genital, anorectal or oropharyngeal NG positivity were observed. A total of 108 patients were NG-positive at two or three anatomical sites (90 MSM and 18 women who reported anal sex or symptoms and who were systematically tested on all three anatomical sites). The remaining amount of sample material was not sufficient for typing for 10 patients (nine MSM and one woman) and therefore these patients were excluded, leaving 98 patients with paired samples for analysis. In total, 205 Cobas NAAT clinical samples were included: 57 urine, 17 vaginal, 92 anorectal and 39 oropharyngeal samples. With these samples, we assessed the clinical application of the culture-free NG-MAST method and the presence of distinct STs within a patient. Data on culture success were retrieved by routine diagnostics because NG culture is mostly performed as part of the national NG resistance surveillance since NAAT diagnosis of NG is the primary diagnostic procedure. All patients were treated with a single dose of ceftriaxone, the primary choice of treatment because no resistance exists in the Netherlands [4]. An additional swab or urine sample for routine NG culture is taken at the treatment visit at the STI clinic only when treatment has not already been provided at the diagnostic visit based on symptoms. For this study, data of this routine culture was available for all patients if culture was performed.

\section{DNA isolation clinical samples}

Total DNA was isolated from $400 \mu \mathrm{L}$ Cobas 4800 clinical samples using the QIAamp DNA Mini Kit (Qiagen, Hilden, Germany) and eluted in $50 \mu \mathrm{L}$ Milli-Q water (MQ). To increase elution yield, we extended the incubation time to $10 \mathrm{~min}$. The eluate was stored at $-20^{\circ} \mathrm{C}$.

\section{DNA isolation cultured gonoccocal and non-gonoccocal Neisseria strains}

Gonoccocal and non-gonoccocal clinical and reference Neisseria strains were inoculated on chocolate agar with IsoVitaleX or blood agar (BectonDickinson, Sparks, United States (US)) and incubated over night at $37^{\circ} \mathrm{C}$ in $5 \% \mathrm{CO}_{2}$. Morphology of the colonies was checked and a single colony was subcultured before DNA isolation. Bacterial suspensions were prepared in sterile saline solution from two or three colonies (depending on the size of the colonies) picked with a pre-wetted sterile swab. The bacteria were pelleted by centrifugation at 2,000 $\mathrm{g}$ for $5 \mathrm{~min}$ and washed once. The pellet was resuspended in $500 \mu \mathrm{L} M Q$ and boiled for $10 \mathrm{~min}$. Cell debris was pelleted by centrifugation at $8,000 \mathrm{~g}$ for $2 \mathrm{~min}$ and the supernatant was stored at $-20^{\circ} \mathrm{C}$. 
TABLE 1

Overview of primers used in PCR and sequencing reactions for NG-MAST and culture-free NG-MAST

\begin{tabular}{|c|c|c|}
\hline & NG-MAST [ ] & Culture-free NG-MAST \\
\hline \multicolumn{3}{|c|}{$\mathrm{PCR}$ primers por $B$} \\
\hline Forward & 5'-CAA GAA GAC CTC GGC AA-3' & 5'-GTT AAT CCG CTA TAA CCC CC-3' \\
\hline Reverse & 5'-CCG ACA ACC ACT TGG T-3' & 5'-CCG ACA ACC ACT TGG T-3' \\
\hline \multicolumn{3}{|c|}{ PCR primers $t b p B$} \\
\hline Forward & 5'-CGT TGT CGG CAG CGC GAA AAC-3' & 5'-TTC CTT CCA AAA AAC CGG AAG CCC G-3' \\
\hline Reverse & 5'-TTC ATC GGT GCG CTC GCC TTG-3' & 5'-CAT TGC CCG GAT AGG CAA ACC A-3' \\
\hline \multicolumn{3}{|c|}{ Sequence primers por $B$} \\
\hline Forward & 5'-CAA GAA GAC CTC GGC AA-3' & 5'-CAA GAA GAC CTC GGC AA-3' \\
\hline Reverse & 5'-CCG ACA ACC ACT TGG T-3' & 5'-CCG ACA ACC ACT TGG T-3' \\
\hline \multicolumn{3}{|c|}{ Sequence primers $t b p B$} \\
\hline Forward & 5'-CGT TGT CGG CAG CGC GAA AAC-3' & 5'-CGT TGT CGG CAG CGC GAA AAC-3' \\
\hline Reverse & 5'-TTC ATC GGT GCG CTC GCC TTG-3' & 5'-TTC ATC GGT GCG CTC GCC TTG-3', \\
\hline
\end{tabular}

\section{NG-MAST genotyping}

PCR for both targets was performed in $50 \mu \mathrm{L}$ reaction volumes using the Biometra T3000 Thermal Cycler (Labrepco Inc., US). Each reaction per target (porB and $t b p B$ ) contained 50 pmol of the NG-MAST forward and reverse primer for the respective target (Table 1), 2.5 U HotStar polymerase (Qiagen), 1× Qiagen PCR buffer, $0.2 \mathrm{mmol} / \mathrm{L}$ dNTP, $5 \mu \mathrm{L}$ DNA lysate and MQ to a volume of $50 \mu \mathrm{L}$. The PCR protocol of Martin et al. was used to amplify the targets but cycles were increased to 30 [7].

The amplicons were precipitated with $50 \mu \mathrm{L} 20 \%$ polyethylene glycol 8000 and $2.5 \mathrm{~mol} / \mathrm{L}$ sodium chloride at $37^{\circ} \mathrm{C}$ for $15 \mathrm{~min}$. Precipitated amplicons were centrifuged at $15,000 \times \mathrm{g}$ for $15 \mathrm{~min}$ and washed twice with ice-cold $80 \%$ ethanol. The pellet was allowed to dry and resuspended in $25 \mu \mathrm{L} \mathrm{MQ}$.

The por $B$ and $t b p B$ fragments were sequenced with their respective forward and reverse primer using the BigDye Terminator v1.1 Cycle Sequencing kit (Thermo Fisher Scientific, Waltham, Massachusetts, US). The sequence protocol has an initial denaturation step of $1 \mathrm{~min}$ at $96^{\circ} \mathrm{C}$, followed by 25 cycles of $10 \mathrm{~s}$ at $96^{\circ} \mathrm{C}$, $10 \mathrm{~S}$ at $55^{\circ} \mathrm{C}($ por $B)$ or $65^{\circ} \mathrm{C}(\operatorname{tbp} B)$, and $3 \mathrm{~min}$ at $60^{\circ} \mathrm{C}$.

\section{Primer design culture-free NG-MAST}

The genome sequences of all NG reference strains published by the WHO $(n=14)$ were downloaded from GenBank and used for multiple alignments with Clustal Omega [19]. A $2 \mathrm{~kb}$ flanking region of the aligned NG-MAST porB and tbpB primers were selected to identify conserved regions. Each flanking region was aligned and conserved regions were tested for in silico specificity using basic-local alignment search tool
(BLAST). A specific sequence was identified that could be used as the forward primer for porB but no specific sequence was identified for the reverse primer, therefore the NG-MAST reverse primer was used which resulted in a fragment of ca $1.2 \mathrm{~kb}$ (Table 1 ). Two specific sequences were identified for $t b p B$ which could be used as a forward and reverse primer, resulting in a fragment of ca $1.8 \mathrm{~kb}$ (Table 1 ).

\section{Culture-free NG-MAST genotyping}

This method was similar to the NG-MAST method apart from the initial PCR. Each reaction per target (por $B$ and $t b p B$ ) contained $50 \mathrm{pmol}$ of the culture-free NG-MAST forward and reverse primer for the respective target (Table 1), 0.2 $\mu \mathrm{L}$ AccuPrime Taq DNA Polymerase High Fidelity (Thermo Fisher), 1× AccuPrime PCR buffer II, $15 \mu \mathrm{L}$ DNA isolated from a clinical sample and MQ to a volume of $50 \mu \mathrm{L}$. The PCR protocol had an initial denaturation step of $5 \mathrm{~min}$ at $95^{\circ} \mathrm{C}$, followed by 40 cycles of $30 \mathrm{~S}$ at $95^{\circ} \mathrm{C}, 60 \mathrm{~S}$ at $58^{\circ} \mathrm{C}$ (porB) or $69^{\circ} \mathrm{C}$ $(t b p B), 2.5 \mathrm{~min}$ at $68^{\circ} \mathrm{C}$, and a final extension of $10 \mathrm{~min}$ at $68^{\circ} \mathrm{C}$. The por $B$ and $t b p B$ amplicons were sequenced with NG-MAST primers (Table 1). The culture-free method was therefore compatible with the NG-MAST online database because we characterised the same fragments of porBand $t b p B$ genes.

\section{Technical validation of culture-free NG-MAST method}

Analytical sensitivity was determined using dilution series ranging from $1.3 \times 10^{6}$ to $1.3 \times 10^{2}$ colony-forming units (CFU) $/ \mathrm{mL}$. Concordance of culture-free NG-MAST method with NG-MAST was tested with seven randomly selected isolates cultured from four urine samples, two anorectal swabs and one oropharyngeal swab, and their respective unculturable Cobas 4800 screening samples 
between January and March 2017. The isolates were subjected to NG-MAST genotyping whereas the clinical samples were subjected to the culture-free NG-MAST method. The analytical specificity was tested with a panel of 32 non-gonococcal Neisseria species strains, including $N$. cinerea $(\mathrm{n}=1), N$. denitrificans $(\mathrm{n}=1), N$. elongata $(n=1), N$. flavescens $(n=1), N$. lactamica $(\mathrm{n}=2), N$. meningitidis $(\mathrm{n}=3), N$. mucosa $(\mathrm{n}=7), N$. perflava $(n=1), N$. polysaccharea $(n=1)$ and $N$. subflava $(\mathrm{n}=14)$.

\section{Data analysis}

The trace files were assembled, trimmed and edited using Bionumerics (version 7.6, Applied Maths, SintMartens-Latem, Belgium). The starting trimming patterns for $p o r B$ and $t b p B$ and lengths were used as described in Martin et al. [7]. Alleles and ST were called according to the NG-MAST online curated database. Phylogenetic trees of porB, tbpBand concatenated sequences were constructed using multiple alignment and unweighted pair group method with arithmic mean (UPGMA) clustering using default settings with gap penalty at $100 \%$.

\section{Ethical statement}

The study protocol was approved as a scientific study not done in humans by the Medical Ethical Committee of Maastricht University Medical Centre (MUMC+; number METC 2017-2-0251) as it concerned a laboratory and observational study using anonymous data and leftover diagnostic samples only. This was part of an STI clinic procedure where patients did not object to the use of their data and samples anonymously for research purposes.

\section{Results}

Analytical sensitivity, specificity and concordance of culture-free NG-MAST

Dilution series in triplicate showed that culturefree NG-MAST consistently yielded PCR products for both porB and $t b p B$ in samples with a $C q$ value $<35$. None of the tested 32 non-gonococcal Neisseria strains were PCR-positive for either por $B$ or $t b p B$ in the culturefree NG-MAST PCR reactions. The seven randomly selected cultured isolates had identical ST as their respective unculturable Cobas 4800 screening sample but distinct ST were observed between the selected isolates.

\section{Paired clinical samples}

In total, $90.2 \%(185 / 205)$ of the selected paired clinical samples were successfully genotyped with the culture-free NG-MAST method. The porB fragment was successfully sequenced in $95.6 \%(196 / 205)$ of samples and $t b p B$ in $93.7 \%(192 / 205)$. Failure of both targets in a sample does not appear to be related to the $\mathrm{Cq}$ value because both low (130) and higher (30-35) Cq values show comparable failure rates (data not shown). We observed 36 different por $B$ and 22 tbpB alleles, resulting in $45 \mathrm{ST}$. Among the samples, por $B-1808$ and $t b p B-29$ were the most common alleles, present in 51 and 49 samples, respectively. Furthermore, we found five previously unidentified $p o r B$ and two tbpB alleles which all had the highest identity with NG using a BLAST search. The most prevalent ST were ST2992 $(n=36)$, ST11461 $(n=30)$, and ST5441 $(n=26)$, and 15 new STs were found.

Routine culture was performed for 95 of the 205 paired clinical samples and 59 (62.1\%) were culture-positive. Typically, only one anatomical site was sampled for culture, and the majority of the culture-positive samples were collected from the genital site (44/59). Culturefree NG-MAST applied to the non-culturable clinical material (Cobas 4800 sample material) of samples send in for culture (including culture-negative samples) showed that $93.7 \%$ (89/95) were genotyped successfully. However, four samples negative in culture-free NG-MAST were culture-positive. Of the remaining 110 uncultured clinical samples, 98 (89.1\%) could be genotyped.

Sequence diversity within por B and $t b p \mathrm{~B}$ alleles High sequence diversity was observed for both porB and tbpB in this study population (Supplement Figures $\mathrm{S}_{1}$ and $\mathrm{S}_{2}$ ). Two porB alleles (90 and 2723) were divergent, with more than 50\% dissimilarity, from all other observed alleles. The newly identified $t b p B$ allele with $91 \%$ similarity with $t b p B$ 1251 was divergent from all other observed alleles with more than $60 \%$ dissimilarity. In addition, the average dissimilarity between $t b p B$ alleles appeared to be greater than between por $B$ alleles.

\section{Sequence types of samples from separate anatomical sites in a patient}

In this dataset of clinical samples, we genotyped 169 paired samples (taken from a single patient at separate anatomical sites) from 80 patients (66 MSM and 14 women) (Supplement Table). We observed distinct concurrent ST in a quarter (20/80) of the patients. They had the following combinations of sample material: urine-anorectal $(n=6)$, urine-oropharyngeal $(n=1)$, anorectal-oropharyngeal $(n=8)$, urine-anorectal-oropharyngeal $(n=1)$, vaginal-anorectal $(n=3)$, and vaginal-anorectal-oropharyngeal $(n=1)$ (Table 2$)$. Similar proportions of distinct concurrent ST were observed in MSM (16/66) and women (4/14). Interestingly, a single patient (patient 32) was NG-positive with a distinct NG strain at all three tested anatomical sites (Table 2 ).

The Figure presents the dissimilarity of concatenated sequences of por $B$ and $t b p B$ between STs. For the majority of the patients with distinct concurrent STs, a large (>15\%) dissimilarity was observed between the concatenated sequences. Patients 31 and 48 had only $1 \%$ dissimilarity between the concatenated sequences. In both patients, the $t b p B$ allele was identical between the distinct ST but the porB allele showed $>1 \%$ dissimilarity, meaning the two ST did not belong to the same genogroup. When assigning ST to genogroups, 
TABLE 2

Characteristics of patients with concurrent Neisseria gonorrhoeae infection with distinct sequence types, including age, risk group, multiple sequence types and NG-MAST results per sample site, the Netherlands, January 2012-May 2016 (n = 42)

\begin{tabular}{|c|c|c|c|c|c|c|c|c|c|c|c|c|c|c|}
\hline \multirow{2}{*}{ Patient } & \multirow{2}{*}{$\begin{array}{c}\text { Age } \\
\text { (years) }\end{array}$} & \multirow{2}{*}{ Risk group } & \multicolumn{3}{|c|}{ Urine } & \multicolumn{3}{|c|}{ Vaginal } & \multicolumn{3}{|c|}{ Anorectal } & \multicolumn{3}{|c|}{ Oropharyngeal } \\
\hline & & & porB & tbpB & ST & porB & tbpB & ST & porB & tbpB & ST & porB & tbpB & ST \\
\hline 9 & 26 & Women $^{\mathrm{a}}$ & - & - & - & 1808 & $91 \%-1251$ & $\begin{array}{c}\text { New } \\
\text { ST1 }\end{array}$ & 1808 & 29 & 2992 & - & - & - \\
\hline 10 & 22 & MSM & - & - & - & - & - & - & 3031 & 33 & 4995 & 1489 & 33 & 10257 \\
\hline 24 & 27 & MSM & - & - & - & - & - & - & 7272 & 33 & New ST4 & $99 \%-7988$ & 110 & $\begin{array}{c}\text { New } \\
\mathrm{ST}_{3}\end{array}$ \\
\hline 27 & 24 & Women $^{\mathrm{a}}$ & - & - & - & 301 & 29 & 359 & 2723 & 110 & 4431 & 301 & 29 & 359 \\
\hline 28 & 20 & MSM & - & - & - & - & - & - & 908 & 27 & 3588 & 2723 & 27 & 15046 \\
\hline 29 & 41 & MSM & 6720 & 188 & 11461 & - & - & - & 1808 & 137 & 11084 & - & - & - \\
\hline 31 & 44 & MSM & 7988 & 110 & 13902 & - & - & - & 908 & 110 & 1407 & - & - & - \\
\hline 32 & 42 & MSM & 182 & 74 & 1247 & - & - & - & 1808 & 29 & 2992 & 1808 & 836 & $\begin{array}{c}\text { New } \\
\text { ST6 }\end{array}$ \\
\hline 33 & 21 & Women $^{a}$ & - & - & - & $\begin{array}{l}99 \%- \\
6405 \\
\end{array}$ & 74 & $\begin{array}{l}\text { New } \\
\text { ST7 }\end{array}$ & 6720 & 188 & 11461 & - & - & - \\
\hline 37 & 25 & MSM & 1808 & 188 & $\begin{array}{l}\text { New } \\
\text { ST8 }\end{array}$ & - & - & - & 182 & 74 & 1247 & - & - & - \\
\hline 39 & 20 & MSM & - & - & - & - & - & - & 3031 & 33 & 4995 & 4288 & 4 & 9382 \\
\hline 48 & 21 & MSM & - & - & - & - & - & - & 6720 & 188 & 11461 & 4288 & 188 & $\begin{array}{c}\text { New } \\
\text { ST9 }\end{array}$ \\
\hline 53 & 41 & MSM & 30 & 18 & 5441 & - & - & - & 3059 & 29 & 5049 & - & - & - \\
\hline 54 & 25 & MSM & - & - & - & - & - & - & 1808 & 29 & 2992 & 30 & 18 & 5441 \\
\hline 55 & 36 & Women $^{\mathrm{a}}$ & - & - & - & 1808 & 29 & 2992 & 6720 & 188 & 11461 & - & - & - \\
\hline 58 & 49 & MSM & 30 & 18 & 5441 & - & - & - & - & - & - & 1808 & 29 & 2992 \\
\hline 59 & 26 & MSM & - & - & - & - & - & - & 1808 & 29 & 2992 & 30 & 18 & 5441 \\
\hline 60 & 42 & MSM & 30 & 18 & 5441 & - & - & - & 1808 & 29 & 2992 & - & - & - \\
\hline 79 & 20 & MSM & - & - & - & - & - & - & 4199 & 29 & $\begin{array}{l}\text { New } \\
\text { ST14 }^{2}\end{array}$ & 4199 & 4 & $\begin{array}{l}\mathrm{New} \\
\mathrm{ST} 12\end{array}$ \\
\hline 80 & 19 & MSM & 6720 & 188 & 11461 & - & - & - & 30 & 29 & 298 & - & - & - \\
\hline
\end{tabular}

NG-MAST: Neisseria gonorrhoeae multi-antigen sequence typing; ST: sequence type.

a Women reporting anal sex or symptoms.

Sample material that was NG-negative or not sampled as part of routine diagnostics is indicated with a hyphen.

we identified two genogroups that consisted of more than five samples: G2992 ( $n=32)$ and G11084 $(n=6)$. Furthermore, we identified three samples belonging to $\mathrm{G}_{1407}$ (one ST1407 and two ST2212) of which only one (ST2212) was culture-positive and susceptible for ceftriaxone.

\section{Discussion}

In this study we show that the culture-free NG-MAST method can readily be used to genotype NG in clinical samples including extra-genital samples. In addition, the method is compatible with the online NG-MAST database. The culture-free NG-MAST method was technically validated by assessing the NG analytical specificity using non-gonoccocal Neisseria species; it demonstrated good specificity for NG as no crossreactivity was observed. Furthermore, concordance with NG-MAST was demonstrated by comparing typing results of non-culturable clinical samples (Cobas 4800 sample material) with cultured isolates, which were taken less than 2 weeks apart. In this time frame, identical genotypes were expected based on the study by Martin et al. [7]. With culture-free NG-MAST, we genotyped $90 \%$ of the selected paired clinical samples with sufficient bacterial load (Cq value 335 ). Extrapolating this genotyping rate to all NG positive samples $(n=1,110)$ would result in successful typing of $56 \%(624 / 1,110)$ of all NG-positive samples. Among all samples sent in for culture, culture-free NG-MAST showed a higher typing rate of $94 \%$ (89/95) compared with the culture-dependent method with $62 \%$ (59/95). However, four of the 59 culture-positive samples were negative in culture-free NG-MAST. The clinical samples testing negative in culture-free NG-MAST could be caused by PCR-inhibitory substances in the clinical material. The majority of the culture-positive samples were collected from the genital site (44/59); that could be explained by the sampling strategy, but the low sensitivity of extra-genital NG culture could also have contributed [2]. This highlights the importance of culture-free genotyping as the current surveillance data would be biased towards genital samples. With 


\section{FIGURE}

Dendogram constructed by multiple alignment of concatenated por $B$ and $t b p B$ sequences clustered with unweighted pair group method with arithmic mean (UPGMA) algorithm, the Netherlands, January 2012-May $2016(\mathrm{n}=169)$

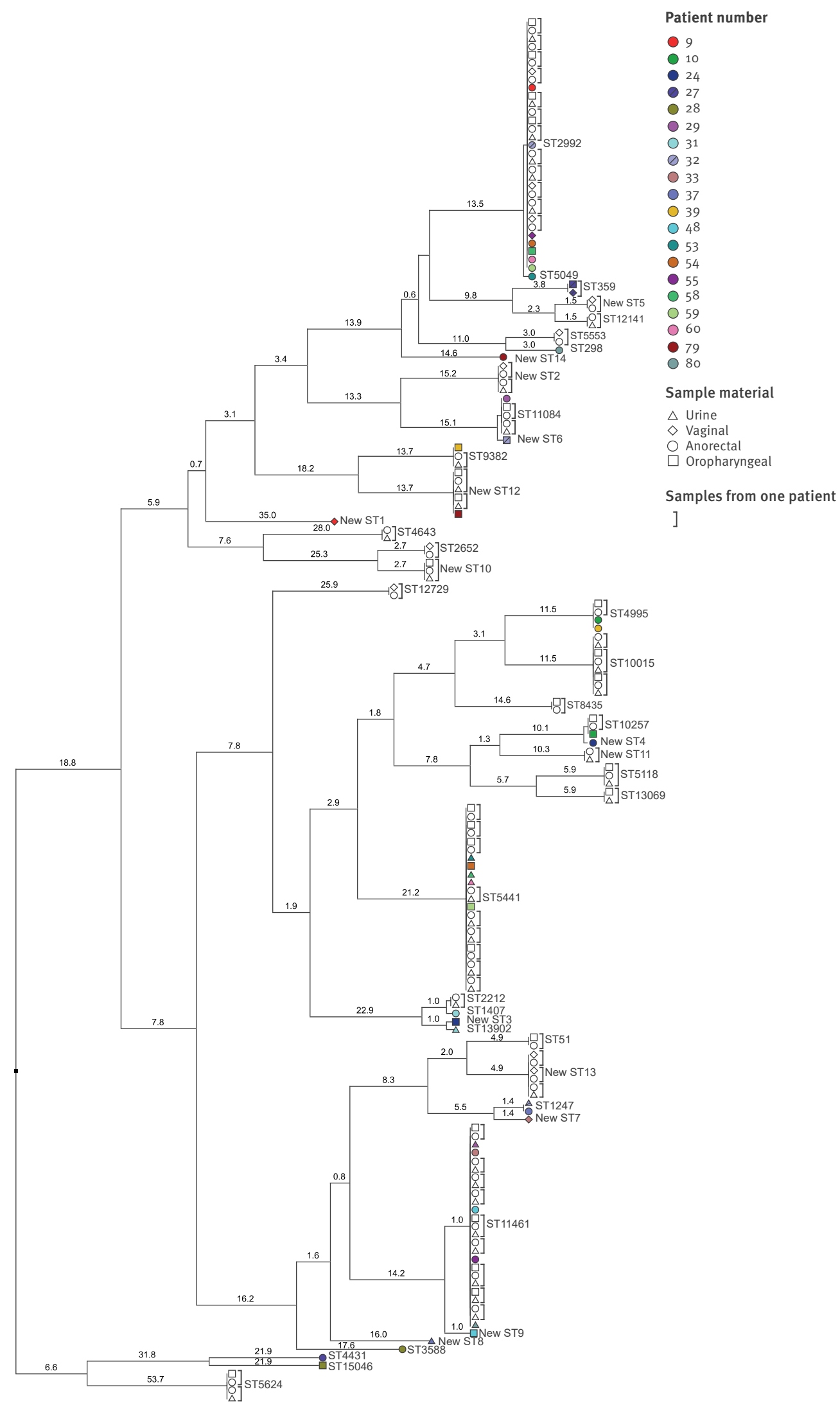

Sequence type (ST) of each sample is shown at the tips of the dendogram and samples of patients with distinct ST are coloured. The branch length depicts the percentage of dissimilarity. 
our method, we were able to genotype 33 of the 36 culture-negative samples, which were mainly extragenital samples (25/33).

Our results show that both sexes were frequently infected with distinct NG strains in a quarter of patients (20/80) which is higher than most previous studies $[6,11,17,18]$. The studies of Whiley et al. (0/4) and Carannante et al. (1/8) assessed, respectively, only four and eight patients with paired samples, which could explain the lower proportion $[6,11]$. Pond et al. (3/71) developed a real-time PCR assay to predict ciprofloxacin resistance with the detection of a resistance-associated SNP [17]. This method uses a single position to identify distinct strains of NG, leading to a lower resolution than NG-MAST where two internal fragments (490 bp and $390 \mathrm{bp}$ ) of highly polymorphic genes are analysed. De Silva et al. (26/206) performed whole genome sequencing only on cultured strains and therefore may have missed distinct strains from samples that were NAAT-positive but culture-negative [18]. A higher percentage of distinct strains in paired clinical samples was reported in a study by Kolader et al. (52/130) which applied por-oparestriction fragment length polymorphism typing [16]. The authors hypothesised that the observed high frequency could be the result of high-risk sexual behaviour or also of recombination in the opa genes.

High-risk behaviour and sex with multiple sex partners on the same occasion may explain the frequently observed distinct ST in our study as we included MSM and women reporting anal sex or symptoms attending our STI clinic, who are considered as risk groups $[1,16]$. Another possible reason could be DNA exchange with commensal Neisseriaspecies or other NG strains $[11,16]$. Patients colonised with multiple NG strains could have different AMR profiles, potentially resulting in under-treatment which could allow dissemination of resistant strains $[6,17]$. However, the impact of multiple strain infections on treatment needs to be addressed in future research to answer questions of the effect on AMR development and dissemination of resistant strains.

The observed concurrent infections with distinct strains in our study would be overlooked in routine diagnostics as Dutch and European (European Centre for Disease Prevention and Control) NG resistance surveillance guidelines recommend culture of only one anatomical site $[2,20,21]$. Without typing data for concurrent NG infections, surveillance data are incomplete and potential transmission links or associations between ST and AMR can be missed. This potentially results in dissemination of unrecognised resistant types. Therefore, early detection and improved surveillance of ST that are linked to AMR could minimise sequelae and prevent dissemination of multidrug-resistant strains.

We observed high variability in both alleles and ST in our study population, which could be due to sampling over a prolonged time period and from different risk groups (MSM and women reporting anal sex or symptoms). For example, ST2212, ST2992, ST5441 and ST5793 are more prevalent in MSM than heterosexual men or women $[10,22,23]$. In our study, these STs were mainly found in MSM, but eight samples with ST2992 and one with ST5793 were from women. Interestingly, we found three samples which belong to the genogroup G1407 (ST1407 ( $=1$ ) and ST2212 ( $=2)$ ) linked to decreased susceptibility to the last first-line treatment with ceftriaxone $[10,23]$. Only one of the three could be cultured (ST2212) and was susceptible to ceftriaxone. Furthermore, ST359, ST2992, ST3588 and ST4995 are linked to azithromycin resistance which is the recommended dual-therapy treatment with ceftriaxone in case of a Chlamydia trachomatis co-infection [1,23-25]. In addition, this dual therapy is applied to slow down emerging resistance or where local resistance data are not available $[1,5,20]$. In the Netherlands, a single treatment with ceftriaxone is applied because no resistance has yet been found in the Netherlands [4]. However, a multidrug-resistant isolate was recently found in the United Kingdom that showed high-level resistance to both ceftriaxone and azithromycin, thereby highlighting the need for improved surveillance [26]. In our study population, we found a high prevalence of ST belonging to genogroup G2992 (19.5\%), which is in line with earlier data from the Netherlands (16.1\%), while genogroups $\mathrm{G}_{1407}(1.7 \%)$ and $\mathrm{G} 359$ (1.1\%) were less frequent (respectively $7.7 \%$ and $6.3 \%$ in the study of Wind et al.) [23]. The genogroup G2992 is also frequently observed in most other countries in Europe [27] and G1407 prevalence is higher in most European countries than in the Netherlands.

Even though many NG-MAST ST are linked to resistance profiles in NG, this does not necessarily imply that the strain is phenotypically resistant $[7,10]$. Additional tests that can identify mutations leading to resistance, for example azithromycin resistance, could give more insight into those strains that cannot be cultured [28]. A limitation of this study is that we only included samples with a higher NG load (Cq value <35); therefore the typing rate of samples with a lower bacterial load is unknown. However, as culture success is also associated with bacterial load, culture-dependent methods are expected to perform worse than our culture-free method in samples with a low NG load (Cq value $\geq 35$ ). This hypothesis is strengthened because only $10 \%$ $(18 / 188)$ of the samples with a $\mathrm{Cq}$ value $\geq 35$ were culture-positive in routine diagnostics. A nested PCR approach might improve genotyping of samples with a low bacterial load as has been applied for medico legal purposes to allow typing from a piece of clothing [29].

\section{Conclusion}

The culture-free NG-MAST method can genotype NG from most non-culturable clinical samples, including extra-genital samples, as cross-reactivity with commensal Neisseria species was not observed. Compared with culture-dependent NG-MAST, culture-free 
NG-MAST has a higher typing rate and does not have high demands on sample conditions. Applying culturefree NG-MAST to clinical samples revealed frequent concurrent infections with distinct ST at separate anatomical sites in MSM and women reporting anal sex or symptoms. These distinct concurrent ST and extragenital NG infections would be missed in the current European surveillance strategy possibly allowing dissemination of resistant NG strains. Including non-culturable and concurrent NG infections in surveillance informs actions to contain the dissemination of multidrug-resistant NG strains.

\section{Conflict of interest}

None declared.

\section{Authors' contributions}

Conceptualisation: PW, CH, LvA. Formal analysis: BvdV, PW, LvA. Methodology: BvdV, PW, LvA. Supervision: PW, $\mathrm{CH}$, NDM, LvA. Validation: BvdV, PW. Writing - Original draft: BvdV. Writing - Review and editing: PW, CH, NDM, LvA.

\section{References}

1. World Health Organization (WHO). Global action plan to control the spread and impact of antimicrobial resistance in Neisseria gonorrhoeae. Geneva: WHO; 2012. Available from: http://apps. who.int/iris/bitstream/handle/10665/44863/9789241503501 eng.pdf;jsessionid=DFA2F277BB3490186A525ABFEF62B3E5?s equence $=1$

2. Recommendations for the laboratory-based detection of Chlamydia trachomatis and Neisseria gonorrhoeae--2014. MMWR Recomm Rep. 2014;63(RR-02):1-19.

3. Goire N, Lahra MM, Chen M, Donovan B, Fairley CK, Guy $\mathrm{R}$, et al. Molecular approaches to enhance surveillance of gonococcal antimicrobial resistance. Nat Rev Microbiol. 2014;12(3):223-9. https://doi.org/10.1038/nrmicro3217 PMID: 24509781

4. Visser M, van Aar F, Op de Coul ELM, Slurink IAL, van Wees DA, Hoenderboom BM, et al. Sexually transmitted infections in the Netherlands in 2017. Bilthoven: National Institute for Public Health and Environment; 2018. Available from: https://www. rivm.nl/bibliotheek/rapporten/2018-0012.pdf

5. World Health Organization (WHO). WHO guidelines for the treatment of Neisseria gonorrhoeae. Geneva: WHO; 2016. Available from: http://apps.who.int/iris/bitstream/hand le/10665/246114/9789241549691-eng.pdf?sequence $=1$

6. Carannante A, Ghisetti V, Dal Conte I, Gregori G, Stella ML, Vacca P, et al. Molecular characterization of Neisseria gonorrhoeae on non-cultured specimens from multiple anatomic sites. Ann Ist Super Sanita. 2017;53(3):213-7. PMID: 28956800

7. Martin IM, Ison CA, Aanensen DM, Fenton KA, Spratt BG. Rapid sequence-based identification of gonococcal transmission clusters in a large metropolitan area. J Infect Dis. 2004;189(8):1497-505. https://doi.org/10.1086/383047 PMID: 15073688

8. Unemo M, Dillon JA. Review and international recommendation of methods for typing neisseria gonorrhoeae isolates and their implications for improved knowledge of gonococcal epidemiology, treatment, and biology. Clin Microbiol Rev. 2011;24(3):447-58. https://doi.org/10.1128/CMR.00040-10 PMID: 21734242

9. Kwong JC, Gonçalves da Silva A, Dyet K, Williamson DA, Stinear TP, Howden BP, et al. NGMASTER:in silico multi-antigen sequence typing for Neisseria gonorrhoeae. Microb Genom. 2016;2(8):e000076. PMID: 28348871

10. Chisholm SA, Unemo M, Quaye N, Johansson E, Cole MJ, Ison CA, et al. Molecular epidemiological typing within the European Gonococcal Antimicrobial Resistance Surveillance Programme reveals predominance of a multidrug-resistant clone. Euro Surveill. 2013;18(3):20358. PMID: 23351652
11. Whiley DM, Goire N, Ray ES, Limnios A, Lambert SB, Nissen MD, et al. Neisseria gonorrhoeae multi-antigen sequence typing using non-cultured clinical specimens. Sex Transm Infect. 2010;86(1):51-5. https://doi.org/10.1136/ sti.2009.037689 PMID: 19843535

12. Dukers-Muijrers NH, Schachter J, van Liere GA, Wolffs PF, Hoebe CJ. What is needed to guide testing for anorectal and pharyngeal Chlamydia trachomatis and Neisseria gonorrhoeae in women and men? Evidence and opinion. BMC Infect Dis. 2015;15(1):533. https://doi.org/10.1186/s12879-015-1280-6 PMID: 26576538

13. Chan PA, Janvier M, Alexander NE, Kojic EM, Chapin K. Recommendations for the diagnosis of Neisseria gonorrhoeae and Chlamydia trachomatis, including extra-genital sites. Med Health R I. 2012;95(8):252-4. PMID: 22970467

14. Unemo M, Shafer WM. Antimicrobial resistance in Neisseria gonorrhoeae in the 21st century: past, evolution, and future. Clin Microbiol Rev. 2014;27(3):587-613. https://doi. org/10.1128/CMR.00010-14 PMID: 24982323

15. Bissessor M, Tabrizi SN, Fairley CK, Danielewski J, Whitton B, Bird S, et al. Differing Neisseria gonorrhoeae bacterial loads in the pharynx and rectum in men who have sex with men: implications for gonococcal detection, transmission, and control. J Clin Microbiol. 2011;49(12):4304-6. https://doi. org/10.1128/JCM.05341-11 PMID: 21956992

16. Kolader ME, Dukers NH, van der Bij AK, Dierdorp M, Fennema JS, Coutinho RA, et al. Molecular epidemiology of Neisseria gonorrhoeae in Amsterdam, The Netherlands, shows distinct heterosexual and homosexual networks. J Clin Microbiol. 2006;44(8):2689-97. https://doi.org/10.1128/JCM.02311-05 PMID: 16891479

17. Pond MJ, Hall CL, Miari VF, Cole M, Laing KG, Jagatia $\mathrm{H}$, et al. Accurate detection of Neisseria gonorrhoeae ciprofloxacin susceptibility directly from genital and extragenital clinical samples: towards genotype-guided antimicrobial therapy. J Antimicrob Chemother. 2016;71(4):897-902. https://doi. org/10.1093/jac/dkv432 PMID: 26817487

18. De Silva D, Peters J, Cole K, Cole MJ, Cresswell F, Dean G, et al. Whole-genome sequencing to determine transmission of Neisseria gonorrhoeae: an observational study. Lancet Infect Dis. 2016;16(11):1295-303. https://doi.org/10.1016/S1473 3099(16)30157-8 PMID: 27427203

19. Unemo M, Golparian D, Sánchez-Busó L, Grad Y, Jacobsson S, Ohnishi M, et al. The novel 2016 WHO Neisseria gonorrhoeae reference strains for global quality assurance of laboratory investigations: phenotypic, genetic and reference genome characterization. J Antimicrob Chemother. 2016;71(11):3096 108. https://doi.org/10.1093/jac/dkw288 PMID: 27432602

20. Workowski KA, Bolan GACenters for Disease Control and Prevention. Sexually transmitted diseases treatment guidelines, 2015. MMWR Recomm Rep. 2015;64(RR-03):1-137. PMID: 26042815

21. European Centre for Disease Prevention and Control (ECDC). Gonococcal antimicrobial susceptibility surveillance in Europe 2015. Stockholm: ECDC; 2017 . Available from: https:// ecdc.europa.eu/sites/portal/files/documents/gonococcalantimicrobial-susceptibility-surveillance-Europe-2015.pdf

22. Cheng CW, Li LH, Su CY, Li SY, Yen MY. Changes in the six most common sequence types of Neisseria gonorrhoeae, including ST4378, identified by surveillance of antimicrobial resistance in northern Taiwan from 2006 to 2013. J Microbiol Immunol Infect. 2016;49(5):708-16. https://doi.org/10.1016/j. jmii.2014.08.016 PMID: 25442864

23. Wind CM, Bruisten SM, Schim van der Loeff MF, Dierdorp M, de Vries HJC, van Dam AP. A Case-Control Study of Molecular Epidemiology in Relation to Azithromycin Resistance in Neisseria gonorrhoeae Isolates Collected in Amsterdam, the Netherlands, between 2008 and 2015. Antimicrob Agents Chemother. 2017;61(6):e02374-16. https://doi.org/10.1128/ AAC.02374-16 PMID: 28373191

24. Shigemura K, Osawa K, Miura M, Tanaka K, Arakawa S, Shirakawa T, et al. Azithromycin resistance and its mechanism in Neisseria gonorrhoeae strains in Hyogo, Japan. Antimicrob Agents Chemother. 2015;59(5):2695-9. https://doi. org/10.1128/AAC.04320-14 PMID: 25712352

25. Brunner A, Nemes-Nikodem E, Jeney C, Szabo D, Marschalko M, Karpati S, et al. Emerging azithromycin-resistance among the Neisseria gonorrhoeae strains isolated in Hungary. Ann Clin Microbiol Antimicrob. 2016;15(1):53. https://doi.org/10.1186/ S12941-016-0166-9 PMID: 27646968

26. Eyre DW, Sanderson ND, Lord E, Regisford-Reimmer N, Chau $\mathrm{K}$, Barker L, et al. Gonorrhoea treatment failure caused by a Neisseria gonorrhoeae strain with combined ceftriaxone and high-level azithromycin resistance, England, February 2018. Euro Surveill. 2018;23(27):1800323. https://doi. org/10.2807/1560-7917.ES.2018.23.27.1800323 PMID: 29991383 
27. European Centre for Disease Prevention and Control (ECDC).

Molecular typing of Neisseria gonorrhoeae - a study of 2013

isolates. Stockholm: ECDC; 2018. Available from: https://ecdc. europa.eu/sites/portal/files/documents/Molecular-typing-Ngonorrhoeae-web.pdf

28. Trembizki E, Buckley C, Donovan B, Chen M, Guy R, Kaldor J, et al. Direct real-time PCR-based detection of Neisseria gonorrhoeae $23 \mathrm{~S}$ rRNA mutations associated with azithromycin resistance. J Antimicrob Chemother. 2015;70(12):3244-9. PMID: 26338048

29. Martin IM, Foreman E, Hall V, Nesbitt A, Forster G, Ison CA.

Non-cultural detection and molecular genotyping of Neisseria gonorrhoeae from a piece of clothing. J Med Microbiol.

2007;56(Pt 4):487-90. https://doi.org/10.1099/jmm.0.46956-o PMID: 17374888

\section{License and copyright}

This is an open-access article distributed under the terms of the Creative Commons Attribution (CC BY 4.0) Licence. You may share and adapt the material, but must give appropriate credit to the source, provide a link to the licence, and indicate if changes were made.

This article is copyright of the authors or their affiliated institutions, 2018. 